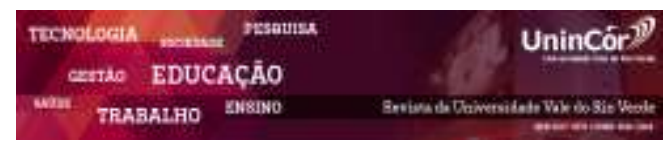

Revista da Universidade Vale do Rio Verde ISSN: 1517-0276 / EISSN: 2236-5362 Vol. 16 | n. 8 | Ano 2018

Camilla Passarela Silva

Faculdade de Medicina de Marília camillapassarelas@gmail.com

Sueli Moreira Pirolo

Faculdade de Medicina de Marília Sueli.moreira@famema.br

\section{ORGANIZAÇÃO DO SERVIÇO PARA O CUIDADO PALIATIVO: REVISÃO BIBLIOGRÁFICA}

\section{RESUMO}

Este estudo teve como objetivo analisar como a produção cientifica tem abordado e conduzido ás pesquisas sobre a organização dos serviços em cuidados paliativos. Trata-se de uma pesquisa descritiva e bibliográfica. Para a busca dos dados foram utilizadas as bases de dados da SCIELO e LILACS, por meio dos descritores: cuidados paliativos, terminalidade $\mathrm{e}$ câncer. Sendo selecionados 33 periódicos publicados no período de 2011 a 2015. A análise dos dados ocorreu por percentual simples para as informações quanto á revista que mais abordou a temática, ao ano de publicação, autor com maior numero de periódicos, categoria profissional do primeiro autor, descritores principais, método empregado na pesquisa. A questão sobre a organização do serviço para realizar os cuidados paliativos foi submetida á análise temática. O tema "serviço organizado em busca da morte digna" foi identificado, o qual foi obtido por meio das categorias analíticas: conceito sobre cuidados paliativos; estrutura para a realização desse cuidado; modo de trabalho a ser realizado; comunicação e educação nessa terapêutica. $\mathrm{O}$ cuidado paliativo deve ser realizado por uma equipe multiprofissional, com a integração das redes de saúde para oportunizar a continuidade do cuidado, e sempre englobar o individuo e sua família no atendimento, oportunizando a comunicação efetiva na relação da equipe com paciente/família. Diante disso conclui-se que a organização do serviço está fundamentada pelo cuidado com enfoque humanístico e requer melhorias para adequar as lacunas existentes acerca da continuidade do cuidado paliativo.

Palavras-chave: Cuidados paliativos na terminalidade da vida. Neoplasias. Serviços de saúde. Qualidade de vida.

\section{ORGANIZATION OF THE SERVICE FOR PALLIATIVE CARE: BIBLIOGRAPHICAL REVIEW}


palliative care was submitted to thematic analysis. The theme "organized service in search of dignified death" was identified, which was obtained through the analytical categories: palliative care concept; structure for this care; mode of work to be performed; communication and education in this therapy. Palliative care should be performed by a multiprofessional team, with the integration of health networks to provide continuity of care, and always include the individual and his family in care, providing effective communication in the team / patient / family relationship. It is concluded that the organization of the service is based on care with a humanistic approach and requires improvements to fit the existing gaps in the continuity of palliative care.

Keywords: Palliative care in the terminality of life. Neoplasms. Health services. Quality of life.

Recebido em: 12/02/2018 - Aprovado em: 28/08/2018 - Disponibilizado em: 15/12/2018

\section{INTRODUÇÂO}

Para Silva (2008), a morte sempre foi e continuará a ser um grande desafio para os profissionais da área da saúde. Expressa uma situação de incurabilidade no cotidiano desses profissionais, os quais sempre questionam como proceder quando não há o que fazer para a cura do doente? Os cuidados paliativos buscam responder a esse questionamento ao ajudar na diminuição do sofrimento de pacientes e familiares com doenças crônicas e terminais, seu enfoque é o paciente em si e não a sua patologia (SANTANA, 2009).

Segundo Santana (2012), esses cuidados iniciaram no século XVII com o movimento hospice, quando foi construídos abrigos para órfãos, pobres e doentes. Já, no século XIX, tiveram características de hospitais, com alas que direcionavam os cuidados a pacientes com tuberculose e câncer. $\mathrm{O}$ cuidado a estes doentes era essencialmente leigo e voltado para o cuidado espiritual e na tentativa do controle da dor.
A Organização Mundial da Saúde (2002) define "Cuidado Paliativo é a abordagem que promove qualidade de vida de pacientes e seus familiares diante de doenças que ameaçam a continuidade da vida, através de prevenção e alívio do sofrimento. Requer a identificação precoce, avaliação e tratamento impecável da dor e outros problemas de natureza física, psicossocial e espiritual". Essa definição traz um enfoque holístico e permite um morrer humanizado.

Espera-se que o cuidado paliativo seja realizado por uma equipe multiprofissional, em trabalho harmônico e convergente, entendendo a pessoa doente “[...] como um ser biográfico, ativo, com direito a informação e a autonomia plena para as decisões a respeito de seu tratamento" (CREMESP, 2008). Tais concepções são recentes na prática profissional e contrapõe a prática vigente centrada no médico e ações curativas. Assim, questiona-se como o serviço de saúde tem viabilizado o cuidado na perspectiva da pessoa que requer cuidado paliativo. 
Também no Brasil, os cuidados paliativos são medidas terapêuticas que irá crescer, visto o aumento de casos de câncer. Segundo o INCA (BRASIL, 2018) estima-se, para o Brasil, a ocorrência de 600 mil casos novos de câncer para o ano de 2018 - 2019, sendo que o câncer de próstata $(68$ mil) em homens e mamas (60 mil) em mulheres serão os mais frequentes. Esse perfil epidemiológico requer atualização e capacitação do profissional da saúde, assim as pesquisas nessa temática podem contribuir a uma prática segura e sem risco para os pacientes e familiares, bem como uma melhor organização do serviço em saúde.

Frente à importância dessa temática para a prática profissional, pergunta-se como a produção cientifica tem abordado e conduzido ás pesquisas sobre a organização dos serviços. Uma vez que, a revisão na literatura permite ampliar nossa compreensão e identificar lacunas de conhecimento e necessidade de novos estudos, bem como aspectos convergentes e divergentes a cerca da organização do serviço para o cuidado paliativo.

\section{OBJETIVO}

Diante desse contexto, o objetivo desta pesquisa foi analisar as contribuições da literatura nacional referente á organização dos serviços para o cuidado paliativo.

\section{MÉTODO}

Este estudo caracteriza-se quanto aos fins como descritivo e quanto aos meios como revisão bibliográfica, a qual permitiu analisar em profundidade as informações a respeito de cuidados paliativos para descobrir as possíveis respostas para o problema eleito. Para tanto, essa pesquisa compreende revisão bibliográfica como investigação que permite captar dados atuais e relevantes sobre determinado tema. Ela se constitui nas seguintes fases: escolha do tema; elaboração do plano de trabalho; identificação; localização; compilação; fichamento; análise e interpretação; redação (MARCONI; LAKATOS, 2010).

Para identificar, localizar e compilar as fontes foram utilizadas as bases de dados da BDENF, SCIELO e LILLACS, no período entre 2011 a 2015. Os descritores utilizados foram: cuidado paliativo, oncologia, neoplasia, terminalidade e câncer. Para a seleção dos dados o critério de inclusão estabelecido foi: texto completo disponível ou não; idioma: português. Nesse estudo utilizaram os critérios de exclusão teses e documento de projeto. Essa seleção permitiu identificar 101 fontes pelo recorte de títulos, após a leitura dos títulos dos artigos foram excluídos os que não preenchiam os critérios deste estudo, sendo identificados 44 artigos. Desses, após a leitura do texto completo, foram excluídos os artigos que não explicitavam as fases métodos científicos e o objetivo da pesquisa com clareza e objetividade ou não respondia a questão estudada, logo, 33 artigos preencheram os critérios propostos e configuram na amostra dessa pesquisa.

A coleta de dados foi elaborada de forma a permitir caracterizar as fontes, seguindo um fichamento contendo na primeira etapa identificação do periódico, o ano de publicação, autor e categoria profissional do 
autor, descritores, idioma, objetivo e método. $\mathrm{Na}$ segunda etapa dados referentes aos recortes feitos nas pesquisas que puderam estabelecer relações entre as informações e os dados obtidos com o problema proposto e analisar a consistência das informações e dados apresentados pelos autores quanto a questão: como o serviço se organiza para realizar os cuidados paliativos?

A análise dos dados ocorreu por percentual simples para as informações de identificação. Para a questão eleita utilizou análise temática, que segundo Minayo (2013), as etapas são: pré-análise, exploração do material ou codificação e tratamento do resultado. Essa análise permitiu identificar o tema "o serviço organizado em busca da morte digna" por meio das categorias analíticas: conceito sobre cuidados paliativos; estrutura para a realização desse cuidado; o modo de trabalho a ser realizado; comunicação e educação nessa terapêutica.

\section{RESULTADOS}

\section{Perfil das fontes}

Observou-se que essa temática foi publicada na Revista de Enfermagem UFRJ com 12,12\% (4 artigos), Revista Ana Nery com 9,09\% (3), Cogitare de Enfermagem 9,09\% (3) e Acta Paulista de Enfermagem 9,09\% (3). Quanto ao período de publicações houve maior número no ano de 2012 com 36,36\% (15) seguido por 30,30\% (10) em 2013.

No que se refere à profissão dos autores, fizeram parte deste estudo 30 autores, a categoria profissional Enfermeiro com $78,78 \%$ (25), sendo que $24,24 \%$ (8) tem doutorado em enfermagem e 18,18\% (6) não apresentaram titulação de categoria profissional.

Os descritores que mais apareceram foram cuidados paliativos $84,84 \% \quad$ (28), enfermagem oncológica $36,36 \%$ (12) e oncologia $21,21 \%$ (7). O tipo de artigo que mais prevaleceu foi artigos originais com $66,66 \%$ (22) e artigo de pesquisa $21,21 \%$ (7).

Em relação aos fins das pesquisas, prevaleceu o descritivo com 48,48\% (16), exploratório com 24,24\% (8). Em relação aos meios $57,57 \%$ (19) utilizaram abordagem qualitativa, $\quad 9,09 \% \quad$ (3) apresentaram abordagem pesquisa de campo, 9,09\% (3) apresentaram abordagem quantitativa. Contudo, 36,36\% (12) não apresentaram no método a natureza da pesquisa.

\section{Serviço organizado em busca da morte digna}

Esse tema selecionado em resposta a questão norteadora, foi obtido por meio de cinco categorias analíticas. A primeira remete ao conceito sobre cuidados paliativos, a segunda aborda a estrutura para a realização desse cuidado, a terceira esta voltada para o modo de trabalho a ser realizado, a quarta é sobre a comunicação e a quinta é sobre educação nessa terapêutica.

Quanto ao conceito de cuidado paliativo, os autores fazem referência à promoção de qualidade de vida para cuidar de pacientes oncológicos em estágio avançado da doença, sem possibilidade da cura. Esses 
cuidados estão baseados em uma visão holística do ser humano, uma "filosofia", um "modo de cuidar", de acordo com os princípios da bioética, de veracidade, respeito, solidariedade e dignidade.

Também, advogam que o cuidado paliativo busca assegurar a vontade e a autonomia das pessoas, valorizar a cultura, crenças, ansiedade e medos e compreender a morte como um processo natural. Esses cuidados configuram em um conjunto de atos multiprofissionais que visa controlar e diminuir todos os sintomas e sofrimentos físico, psicológico, social e espiritual, decorrentes da doença por meio da prevenção, ajudando a família e os cuidadores no processo de luto e suporte na tomada de decisões.

"Cuidados direcionados a pessoa considerada pela ciência médica sem possibilidade da cura, mas que podem ser cuidadas visando assegurar conforto e dignidade no processo de morrer e na morte." (Silva, 2015).

"Cuidado paliativo é uma "filosofia”, um "modo de cuidar" que visa a aumentar a qualidade de vida e pacientes e suas famílias, que enfrentam problemas associados a doenças que ameaçam a vida, tendo como objetivo a prevenção e alívio do sofrimento pelo controle dos sintomas." (Vieira, 2012)

"Apesar da terminalidade de vida estar definitivamente confirmada, o paciente $e$ a família devem receber apoio e acolhimento dos cuidados paliativos, que defende a morte como um processo natural e que proporcionam cuidados que visam controlar e diminuir todos os sintomas decorrentes da doença por meio da prevenção, além de aliviar o sofrimento físico, psicológico, social e espiritual, incluindo o apoio a família e atenção ao luto." (Capello, 2012)

Os autores trazem a estrutura como elemento importante, para a organização do serviço. Comentam sobre a rede de atenção a saúde, as dependências físicas do serviço e finalidade da unidade de cuidados paliativos, a composição da equipe e a atribuição do enfermeiro.

A rede de atenção á saúde deve estar integrada para oportunizar a continuidade dos cuidados entre as diferentes estratégias de assistência à saúde, de forma a oferecer instituições de alta complexidade, unidade de internação, ambulatório, centros de saúde e domicílio para atender a demanda que requer cuidado paliativo. Quanto á dependência de uma unidade ${ }^{-}$de cuidado paliativo foi apresentado um local com seis leitos, com a possibilidade dos pacientes trazerem objetos pessoais para tornar o quarto uma extensão de sua casa. $O$ setor pode oferecer uma sala multiuso, decorada para os usuários. Destaca-se a necessidade de um veículo de médio porte para transporte de pacientes e familiares.

Em relação á finalidade de uma unidade de cuidados paliativos, os pesquisadores afirmam que essa unidade deve atender portadores de agravos oncológicos sem possibilidades terapêuticas de cura e seus 
familiares, de forma individualizada. A unidade deve promover o controle da dor e atenção aos familiares no momento do luto, além de atuar nas dimensões psicológicas, sociais e espirituais, com o intuito de investir na melhoria de qualidade de vida.

Esses autores apresentam a composição da equipe de saúde, que se constituí por médico, enfermeiro, assistentes sociais, nutricionista, psicóloga, fisioterapeuta, capelão, técnica de enfermagem e motorista. Destaca a atribuição do enfermeiro por estar envolvido desde o inicio do tratamento tanto para o controle da dor e demais sinais e sintomas, quanto para ansiedade e depressão, e realizar também o compartilhamento das decisões do cuidar, facilitando a criação de vínculo e acolhimento dos pacientes e familiares.

“A organização dos cuidados paliativos requer coordenação entre as diferentes estratégias de assistência à saúde, principalmente na internação hospitalar, situação em que se encontra a maioria dos pacientes com neoplasias em estágio avançado. Essa coordenação oportuniza a continuidade dos cuidados e o acolhimento dos pacientes $e$ familiares em ambiente domiciliar." (Fripp, 2012)

"A equipe de uma unidade de cuidados paliativos é formada por médicos, enfermeiros e outros profissionais, como fisioterapeutas, psicólogos, assistentes sociais e capelães." (Kappaun, 2013)
"O cuidado de enfermagem envolve o controle da dor e demais sinais e sintomas, da ansiedade e depressão, além do compartilhament das decisões do cuidar com a criança e sua família. A atuação da equipe de enfermagem diante da complexidade do cuidado a essas crianças compreende a necessidade de empenho da equipe de saúde, por meio do trabalho interdisciplinar.” (Mutti, 2012)

“[...] sugeriu a criação do Núcleo de Cuidados Paliativos. [...] com seis leitos, para atendimento a pacientes oncológicos fora de possibilidades terapêuticas de cura, incluindo seus familiares, tem a intenção de oferecer cuidados individualizados, de acordo com as necessidades de cada paciente. Conta com uma equipe multiprofissional preparada, e de um local com maior privacidade, favorecendo o atendimento diferenciado." (Vieira, 2012)

O trabalho em saúde para o cuidado paliativo foi mencionado pelos autores ora organizado por modo equipe de saúde multiprofissional ora interdisciplinar, ora por abordagem multidisciplinar. Pauta-se no atendimento integral, em decisões compartilhadas, em estratégia de coprodução. As fontes trazem a produção de protocolos e rotinas para o serviço e o planejamento da assistência de enfermagem para identificar prioridades do cuidado.

Apresenta a equipe de enfermagem com a proposta de educar, cuidar, promover, advogar e coordenar o seu cuidado. Esse profissional deve ser capaz de participar de 
forma positiva, disposto ao diálogo, ter sensibilidade, respeitar a liberdade e dignidade do ser humano. Manter-se aberto para novas relações de poder entre profissionais e autonomia do usuário. Criar um vínculo com o paciente um atendimento humanizado. Estar atento para as suas próprias necessidades, limites, responsabilidades, deveres e direitos.

"Além disso, evidencia-se a necessidade de o grupo de multiprofissionais atuar de modo interdisciplinar, para atender às diferentes condições de saúde da pessoa que vivencia a sua finitude." (Arrieira, 2011)

"O destaque dado à relação entre equipe de saúde e usuários configura um tipo de estratégia de coprodução, ou seja, o envolvimento conjunto nas operações para prover cuidados e a emergência do usuário como ator pertinente a gestão de muitas das atividades. Parte-se do pressuposto de que a abordagem em cuidados paliativos requer a constituição de uma equipe multiprofissional $e$ a participação ativa do paciente e de seus familiares" (Schinzari, 2013)

"O cuidado de enfermagem ofertado aos pacientes e familiares na oncologia, visa a "[...] prover conforto, agir e reagir adequadamente frente à situação de morte com o doente, família e consigo mesmo; promover o crescimento pessoal do doente, família e de si mesmo, valorizar o sofrimento e as conquistas, empoderar o outro com seu cuidado $e$ empoderar-se pelo cuidado, lutar para preservar a integridade física, moral, emocional e espiritual, conectar-se, vincular-se e auxiliar o outro e a si mesmo a encontrar significados nas situações”. Para que estas medidas se tornem-se mais efetivas, a equipe de enfermagem deve educar, cuidar, promover, advogar e coordenar o seu cuidado." (Silva, 2012)

"Preconizando o cuidado integral e implicando em mudanças nas relações de poder entre profissionais de saúde para horizontais, de maneira que se constituam equipes interdisciplinares, além de melhorar a relação entre profissionais e usuários, para que se desenvolva realmente a autonomia dos usuários. ” (Porto, 2014)

Os autores compreendem que a comunicação consiste em um elemento importante e deve ser priorizado para atender as necessidades dos familiares, paciente $\mathrm{e}$ equipe de saúde. Deve ocorrer por meio de um dialogo aberto e eficaz que permita a interação e inter-relação entre profissional de saúde e o paciente e sua família, na perspectiva da interdisciplinaridade.

O profissional precisa respeitar os desejos do paciente, apresentar toque afetivo, o olhar, o sorriso, a proximidade física e a escuta atentiva, observar a linguagem corporal, atentar para não só ouvir as pessoas, mas também como estão dizendo. Valorizar a comunicação de más notícias e, sobretudo, interrogar a respeito das expectativas e conhecimento do paciente e família sobre seu problema de saúde e tratamento a ser realizado. 
"Durante a interação com os pacientes ou familiares é importante saber escutar ativamente, observar a linguagem corporal, ouvir não só o que as pessoas estão dizendo, mas também como estão dizendo." (Pinto, 2011)

"Para uma comunicação adequada por meio da interação e inter-relação entre profissional de saúde e o paciente e sua família, e necessário valorizar a importância da comunicação de más noticias e, sobretudo, conhecer as estratégias de comunicação; interrogar a respeito das expectativas $e$ conhecimento do paciente e família sobre sua doença e tratamento, afirmar a solicitude e interesse por aspectos multidimensionais do paciente, toque afetivo, o olhar, o sorriso, a proximidade física e a escuta atentiva." (Sanches, 2013)

Os processos educativos sobre cuidados paliativos são valorizados para o preparo dos profissionais de saúde e uma melhor organização do trabalho. A atividade educativa tem como intenção abordar processos de adoecimento e morte, pautados em aspectos humanísticos e éticos. Podendo ser democrática, participativa, problematizadora e transformadora, ou por meio de seminário interdisciplinar a partir de temas.

"Acredita-se que o acesso a conhecimentos e informações sobre cuidados paliativos possam servir a uma melhor organização do trabalho de enfermagem, preparando seus profissionais para prestar mais atenção à qualidade de vida, à vida interior $e$ às relações humanas, numa nova consciência configurada na psicologia, na filosofia, na antropologia e na religião” (Silva, 2012)

"Ressalta-se a importância do aperfeiçoamento dos profissionais enfermeira(o)s no que diz respeito às suas competências e habilidades pedagógicas, visando à efetividade do seu processo educativo, a fim de propiciar maior participação do paciente. Para tanto, esse pro cesso deve contemplar características especiais, tais como: ser democrático, participativo, problematizador e transformador." (Bernardes, 2014)

\section{DISCUSSÃO}

Os artigos contemplam os elementos que Marx (1994 apud Peduzzi 2007, p. 20) ${ }^{1}$ define sobre processo de trabalho, onde para ser eficaz são necessários três elementos primordiais, o objeto, os instrumentos e os agentes, os quais orientam a organização do serviço. O objeto é o que se transforma em produto; os meios e instrumentos são para realizar o trabalho; e os agentes são quem realizam esse trabalho. Em cuidados paliativos o objeto seria os pacientes e seus familiares, os meios e instrumentos são os locais onde pode ocorrer esse tratamento e os recursos necessários a serem utilizados, e os agentes são os profissionais de saúde que realizaram esses cuidados.

\footnotetext{
${ }^{1}$ MARX, K. O capital. 14. ed. São Paulo: Difel, 1994. v. 1, p. 202.
} 
Para os autores, os cuidados paliativos é uma terapêutica voltada para o alívio e sofrimento de pacientes que estão em fase terminal e seus familiares. Em que esses cuidados devem gerar qualidade de vida, respeitando os desejos e valorizando a dignidade do paciente, o que entra em concordância com a definição da Organização Mundial da Sáude (2002). Ressalta que, para que esse cuidado seja realizado de forma eficiente, o serviço tem que possuir uma organização e processo de trabalho que possa suprir todos esses elementos do cuidado integral, sendo realizado por uma equipe multiprofissional com um trabalho interdisciplinar. A equipe multiprofissional é composta por diversas disciplinas, porém com um cuidado fragmentado, já a interdisciplinar é a interação entre duas ou mais disciplinas podendo refletir na organização do serviço e ensino (SAAR; TREVISAN, 2007).

Essa terapêutica pode ser realizada desde o diagnóstico até por todos os estágios da doença. Pois, desde o inicio pode haver alterações em diferentes aspectos no individuo, assim o serviço precisa ampliar a visão do cuidado paliativo para além da hora da morte e incluir a pessoa no atendimento a partir da identificação da patologia, que expresse a impossibilidade terapêutica (SILVA, 2008).

Trazem que o serviço de saúde tem capacidade de avaliar e designar o local onde seu paciente fará seu tratamento, de acordo com o grau de complexidade do estado de saúde. Para isso é necessário um ambiente acolhedor, podendo assemelhar com o domicilio trazendo pertences pessoais. Sobre a estrutura física adequada para os cuidados paliativos não houve clareza de como deve ser, os equipamentos e insumos não foram abordados com evidência pelos autores. Eles citaram diversos ambientes, porém não apresentaram às condições físicas do espaço, os instrumentos utilizados, as tecnologias necessárias.

Trouxeram também sobre a importância da comunicação (verbal ou não verbal) de qualidade. Nesse sentido, a comunicação é essencial na relação entre a equipe e paciente/família. Essa relação facilita a criação do vínculo, a empatia da equipe com o paciente. Para Chavianato (2003), a comunicação é toda troca de informação, tanto verbal, escrito ou não-verbal. Diante da análise dos artigos, houve predomínio da comunicação dialógica, onde deve ser de forma clara e objetiva.

Foi identificado sobre a importância da educação profissional para a qualificação do cuidado, como forma de capacitações e educação continuada. Isso fará com que os profissionais se aperfeiçoam sobre o cuidado prestado, mantendo-se atualizados. Mas não somente a educação continuada é necessária para essa qualificação, á educação permanente também é relevante onde diante de uma necessidade de interação profissional e um trabalho compartilhado.

\section{CONCLUSÃO}

A temática estudada está presente na literatura nacional o que permitiu cumprir com os objetivos previstos. A metodologia 
escolhida favoreceu analisar a produção cientifica sobre cuidado paliativo tendo a organização do serviço como objeto de estudo. O profissional enfermeiro está mais envolvido nas pesquisas desse assunto, contudo $\mathrm{o}$ conceito de cuidado paliativo pressupõe um enfoque multiprofissional para o cuidado. Assim, precisamos incentivar o interesse dos demais profissionais da saúde para a produção cientifica.

Diante dos artigos selecionados foi possível verificar a importância dos cuidados paliativos em pacientes em fase terminal, a maneira de como a família é abordada nas fases do luto de forma individual. A necessidade de uma equipe multiprofissional trabalhando de forma interdisciplinar, realizando um cuidado integral. Trouxeram sobre a relevância da equipe de enfermagem nesse processo, sendo fundamental em todo o cuidado. Dessa forma, o cuidado estará aliviando todos os sinais e sintomas do paciente, seguindo os princípios éticos dos direitos humanos.

Porém, quanto à organização do serviço pouco traz sobre a estrutura física, instrumentos e tecnologias que são necessárias para desenvolver esse cuidado. Há também uma referência para a qualificação dos profissionais. A área da saúde está aos poucos desenvolvendo essa terapêutica, mas ainda requer uma organização de serviço integradora que atenda a demanda por estes cuidados, que possua uma estrutura física e humana capacitada.

\section{REFERÊNCIAS}

BRASIL. Instituto Nacional de Câncer José Alencar Gomes da Silva. Estimativa 2018: incidência de câncer no Brasil. Rio de Janeiro: INCA, 2018. Disponível em: < http://www1.inca.gov.br/inca/Arquivos/estimativa2018.pdf>. Acesso em: 05 jan. 2018.

CARVALHO, R. T.; PARSONS, H. A. Manual de cuidados paliativos ANCP: ampliado e atualizado. 2. ed. São Paulo: Academia Nacional de Cuidados Paliativos, 2012.

CHIAVEANATO, I. Introdução a teoria geral de administração. 7. ed. Rio de Janeiro. Elsevier, 2003.

\section{CONSELHO REGIONAL DE MEDICINA DO ESTADO DE SÃO PAULO. Cuidado paliativo. São Paulo: CREMESP, 2008. \\ FERRAZ, A. P. C. M.; BELHOT, R. V. Taxonomia de Bloom: revisão teórica e apresentação das adequações do instrumento para definição de objetivos instrucionais. Rev. Gestão Produção, São Carlos, v. 17, n. 2, p. 421-431, 2010.}

MARCONI, M. A.; LAKATOS, E. M. Fundamentos de metodologia científica. 7. ed. São Paulo: Atlas, 2010 .

MINAYO, M. C. S. O desafio do conhecimento: pesquisa qualitativa em saúde. 13. ed. São Paulo: Hucitec, 2013.

PEDUZZI, M. Trabalho em equipe de saúde da perspectiva de gerentes de serviços de saúde: possibilidades da prática comunicativa orientada pelas necessidades de saúde dos usuários e população. 2007. 247 f. Tese (Livre docência) - Escola de Enfermagem, Universidade de São Paulo, São Paulo, 2007.

SAAR, SRC, TREVIZAN, MA. Papéis profissionais de uma equipe de saúde: visão de seus integrantes. Rev. Latinoam Enferm., Ribeirão Preto, v. 15, n. 1, p. 106-112, 2007.

SANTANA, J. C. B. et al. Cuidados paliativos aos pacientes terminais: percepção da equipe de enfermagem. Bioethikos, São Paulo, v. 3, n. 1, p. 77$86,2009$.

SILVA, E. P.; SUDIGURSKY, D. Concepções sobre cuidados paliativos: revisão bibliográfica. Acta Paul. Enferm., São Paulo, v. 21, n. 3, p. 504-508, 2008.

WAAL, P.; TELLES, M. Verbos de aplicação para elaboração de objetivos: a taxionomia de Bloom. Ponta Grossa: Universidade Estadual de Ponta Grossa, 2004. Disponível em:

<http://www.uepg.br/formped/disciplinas/Organizacao 
Trabalho/TAXONOMIA\%20DE\%20BLOOM.pdf>. Acesso em: 09 fev. 2018.

WORLD HEATH ORGANIZATION. Definition palliative care. Geneva: WHO, 2002. Disponível em: <http://www.who.int/cancer/palliative/definition/en/>. Acesso em: 09 fev. 2018.

\section{Camilla Passarela Silva}

Enfermeira graduada pela FAMEMA.

Residente de Urgência e Trauma - HRPP.

\section{Sueli Moreira Pirolo}

Doutora em Enfermagem Fundamental pela USP/Ribeirão Preto. 